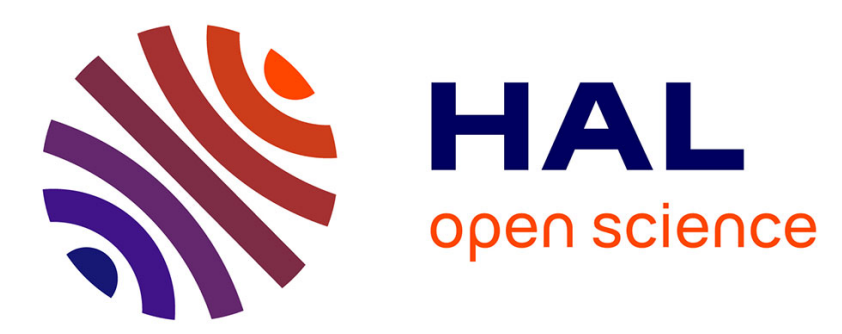

\title{
A probabilistic model to predict the formation and propagation of crack networks in thermal fatigue
}

Nicolas Malesys, Ludovic Vincent, François Hild

\section{To cite this version:}

Nicolas Malesys, Ludovic Vincent, François Hild. A probabilistic model to predict the formation and propagation of crack networks in thermal fatigue. International Journal of Fatigue, 2009, 31 (3), pp.565-574. 10.1016/j.ijfatigue.2008.03.026 . hal-00322191

\section{HAL Id: hal-00322191 \\ https://hal.science/hal-00322191}

Submitted on 16 Sep 2008

HAL is a multi-disciplinary open access archive for the deposit and dissemination of scientific research documents, whether they are published or not. The documents may come from teaching and research institutions in France or abroad, or from public or private research centers.
L'archive ouverte pluridisciplinaire HAL, est destinée au dépôt et à la diffusion de documents scientifiques de niveau recherche, publiés ou non, émanant des établissements d'enseignement et de recherche français ou étrangers, des laboratoires publics ou privés. 


\title{
A probabilistic model to predict the formation and propagation of crack networks in thermal fatigue
}

\author{
Nicolas Malésys, ${ }^{\text {a,b}}$ Ludovic Vincent, ${ }^{\mathrm{a}}$ François Hild ${ }^{\mathrm{b}}$ \\ ${ }^{\mathrm{a}} C E A$ Saclay, DEN-DANS/DMN/SRMA/LC2M, \\ F-91191 Gif sur Yvette cedex, France \\ ${ }^{\mathrm{b}}$ LMT-Cachan, ENS de Cachan, CNRS UMR 8535, Université Paris 6, \\ 61 avenue du Président Wilson, F-94235 Cachan cedex, France
}

\begin{abstract}
A probabilistic model is presented to predict the formation and propagation of crack networks in thermal fatigue. It is based on a random distribution of sites where cracks initiate and on the shielding phenomenon corresponding to the relaxed stress field created around cracks. Currently, the model considers only heterogeneous uniaxial stress loading even if thermal fatigue is multiaxial. However, the first simulations on a uniaxial mechanical loading representative of the stress gradient that appears in thermal fatigue shocks are in qualitative agreement with experimental results. The larger the stress amplitude, the denser the crack network and the smaller the crack sizes.
\end{abstract}

Key words: Crack network, Poisson Point Process, Shielding, Weibull model 


\section{Introduction}

Thermal fatigue is still a major issue in many industrial activities, as proven by very recent publications on this subject in aircraft [1,2], automotive [3,4], metal forming [5-7], braking [8-10], electronic [11,12] and nuclear [13-16] industries. In the case of cooling systems in nuclear power plants, observations revealed the presence of thermal crazing on the internal part of pipes [17]. These crack networks are not a major issue for the structure as long as cracks do not traverse the pipe thickness. Consequently, it is important to know how the crack networks are created and propagate.

The present approach models these phenomena in a probabilistic way, namely the sites where cracks may initiate are described by a Poisson point process. This hypothesis was already used to analyze probabilistic features associated with brittle fracture [18], multiple cracking of composites [19], dynamic fragmentation of ceramics [20], damage and fracture in cyclic fatigue [21-23]. In many instances, Monte-Carlo simulations are then devised to account for the afore-mentioned phenomena $[19,21,24,22]$. In the present case, closed form expressions are derived. The conditions for initiation or propagation of cracks are controlled by the probability of finding these sites in the relaxed stress zone created by the cracks already present on the material surface. It is shown that this shielding phenomenon it the key for understanding the partition between stopped and propagating cracks. The present model gives access to various quantities such as the shielding probability, the propagating and formed crack densities. The first quantity gives an information on the possible saturation of the crack network, the second one describes the number of active cracks per unit surface in the network and the last one represents the total number 
per unit surface of cracks and is thus directly comparable with the densities of crack networks obtained in thermal fatigue experiments (e.g., SPLASH tests [25]).

The SPLASH configuration, derived from Marsh's experiments [26], mimics in laboratory environments the conditions for initiation and development of crack networks due to thermal fatigue in the same way as those observed in the mixing zone of cooling systems of Pressurized Water Reactors (PWR). Other experimental facilities have been developed to produce thermal fatigue on tubular specimens (for instance Cythia [27], Biax [28] and Fat3D [29] at the Atomic Research Center (CEA), or Intherpol [30] at Électricté de France $(\mathrm{EDF}))$. On some of the previous experimental configurations (namely, Biax and Intherpol), a mean axial stress may be superimposed to the cyclic thermal fatigue loading. The advantage of the SPLASH experiment compared with the other ones is its higher frequency that allows for running tests up to one million cycles. The thermal loading is however more difficult to control. A few large scale thermohydraulic tests have been designed to reproduce the mixing conditions in a tee, but the temperature measurements on the surface of the pipe is difficult to perform and the interpretation of such tests requires extensive care.

In the first part of the paper, the framework of the study is presented. Details are given on the formation and propagation of crack networks in thermal fatigue by considering several scales of propagation and by introducing various hypotheses on which the probabilistic model is derived. Second, the applied loading is evaluated and the material parameters are identified. In the last part, the results of calculations associated with the probabilistic model are presented and commented. In particular, the changes of the obscuration prob- 
ability, the mesocrack density and the propagation density with the number of cycles for a loading representative of SPLASH conditions are obtained. A simplified approach of this model is presented by considering a particular crack propagation law. This approach makes it possible to give bounds to all quantities of the probabilistic model. The first results are then compared with those for more severe conditions in order to validate the model in a qualitative way.

\section{Crack Networks}

\subsection{Different Scales}

In high cycle fatigue, three scales are considered [31], namely, the micro-, mesoand macroscales. The microscale corresponds to stage I propagation of cracks in high cycle fatigue and interacts with the material microstructure (e.g., the characteristic length is that of grains for the 304L stainless steel considered herein). During this step, the cracks are considered as microstructurally short (they will be referred to as microcracks). This scale ends when the crack propagation is no longer dependent on the microstructure, which corresponds to the mesoscale or stage II. Mesocracks are formed after microcrack propagation or alternatively, mesocracks are formed on randomly distributed sites and with an initial size $a_{0}=64 \mu m$ greater than the average grain size of, say, 304L stainless steel $\left(\phi_{g}=50 \mu m\right)$. Hence, with such a definition, it is considered that once a mesocrack has formed, it cannot stop on microstructural obstacles such as grain boundaries (contrary to microcracks). We assume that mesocracks propagate according to a Paris' law. The last step is concerned with coalesced mesocracks that form a macrocrack (i.e., a long crack). 
In the present work, we only deal with crack networks formed at the mesoscale (i.e., made of mesocracks) and not crack networks that may appear at the microscale. Coalescence is not described.

\subsection{Crack Obscuration Process}

With these three scales, one needs to model how a mesocrack may form from a microcrack and therefore to model interactions of microcracks with other mesocracks. Considering the stress field around a crack, a stress relaxation zone is observed. In this work, this zone is called obscuration (or shielding) zone. If a microcrack belongs to any of these zones, the stress applied to the microcrack is less than the remote stress, thus the microcrack cannot form a mesocrack. Conversely, if the microcrack is not located in a shielding zone, it forms a mesocrack. Similarly, if a mesocrack falls within the obscuration zone of another mesocrack, it will not be able to further propagate. This phenomenon is called obscuration process. In the present case, this process is binary (i.e., a crack is either completely shielded, or not).

\subsection{Continuous Formation}

An assumption of continuous formation of cracks is made, namely, the microcracks form mesocracks during the course of time but not all at the same time (in the present case, time is equivalent to number of cycles for a constant amplitude and frequency test). Figure 1 shows the development of cracks in a

homogeneous tensile stress field. At the beginning (i.e., when the number of cycles $N$ is equal to 0 ), there are no mesocracks as shown in Figure 1 when 

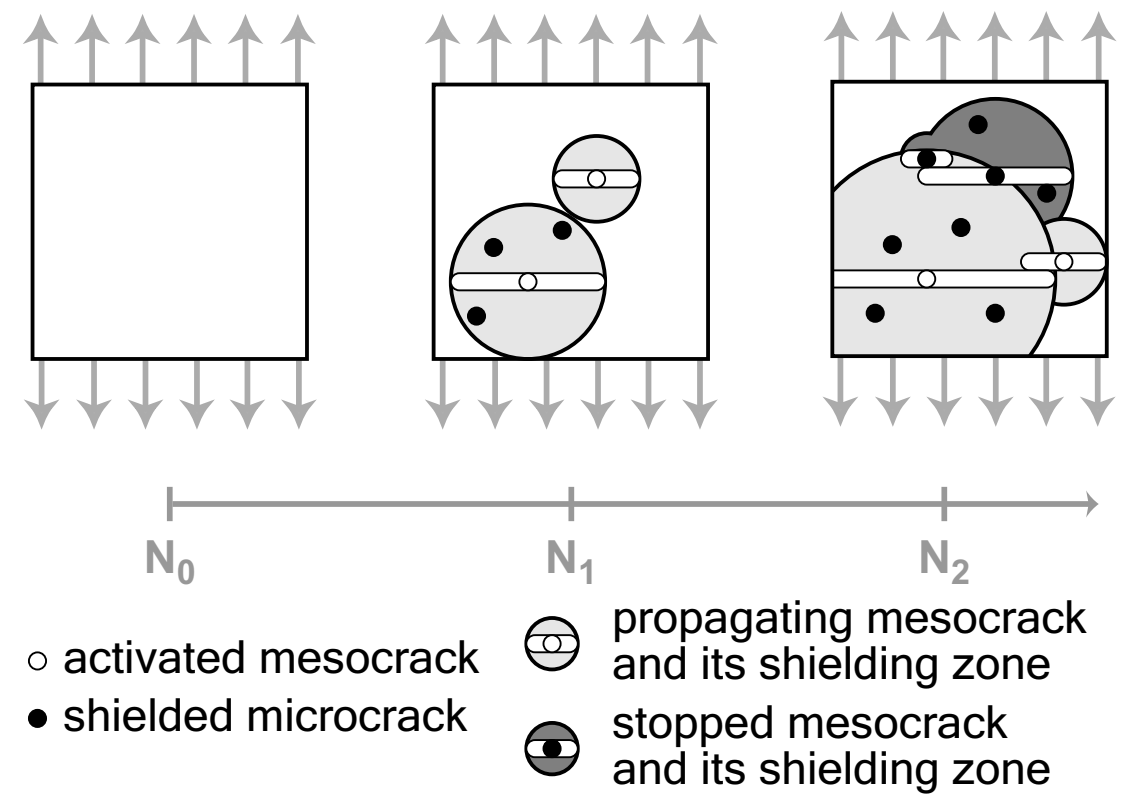

Fig. 1. Depiction of an obscuration process.

$N<N_{0}$. When the number of cycles increases, microcracks are gradually created and they eventually form mesocracks with their corresponding obscuration zone. Consequently, some microcracks that belong to shielding zones do not form new mesocracks. There are also mesocracks that fall into other shielding zones and do not continue to grow (they are stopped). In Figure 1 when $N=N_{1}$, there are two propagating mesocracks with their respective obscuration zone and three shielded microcracks in the shielding zone of the biggest propagating mesocrack. When $N=N_{2}$, the smallest propagating mesocrack of the second step $\left(N=N_{1}\right)$ is now in the shielding zone of the biggest one; it cannot keep on propagating and thus stops. Moreover, a new propagating mesocrack appeared and, in the present case, six microcracks are shielded. If the number of cycles keeps on increasing and if there is only one active crack left (i.e., the largest one), at one stage all the field will be obscured. The weakest link hypothesis [32] then applies, and a Weibull model [33] may be retrieved. 


\subsection{Microcrack Density}

The microstructure of the material is modeled in terms of sites where cracks may be initiated. These sites are approximated by a Poisson point process of intensity $\lambda_{t}$ (i.e., their average number per unit surface or length). A power law is chosen which leads to a Poisson-Weibull model $[19,18]$

$$
\lambda_{t}(\Delta \sigma)=\lambda_{0}\left(\frac{\Delta \sigma}{\sigma_{0}}\right)^{m}
$$

where $m$ is the Weibull modulus (i.e., it characterizes the scatter in local fatigue limits or similarly in local yield stress levels), $\Delta \sigma$ the applied stress variation, and $\Delta \sigma_{0}$ the scale parameter relative to a reference density $\lambda_{0}$ [33]. The considered distribution accounts for microplastic activity close to the material surface [25]. For this type of mechanism, it was shown that the power law (1) is able to model not only the fatigue properties but also dissipation phenomena [23]. As explained in Section 2.3, continuous formation of mesocracks corresponds to an incubation "time" (or number of cycles), that is the time needed by microcracks to initiate and propagate before forming a mesocrack. Moreover, one is only interested in the formation and propagation of mesocracks in order to characterize the crack network. Therefore, an additional parameter is introduced in the formulation of the intensity of the Poisson point process that allows one to limit the intensity of the process to mesocracks.

Let $\lambda_{t I}$ be associated with $\lambda_{t}$ but dependent upon the stress variation $\Delta \sigma$ and the number of cycles $N$

$$
\lambda_{t I}(\Delta \sigma, N)=\lambda_{t}\left[\left\langle\Delta \sigma-\Delta \sigma_{u}(N)\right\rangle\right]
$$


where $\Delta \sigma_{u}(N)$ corresponds to an incubation stress related to the incubation time (see Section 3.2) and where 〈.> Macauley's brackets. Equation (2) shows that the formation process needs a minimum number of cycles $N_{\min }$ (i.e., an incubation time) to form the first mesocrack such that

$$
\Delta \sigma-\Delta \sigma_{u}\left(N_{\min }\right)=0
$$

The continuous formation assumption returns the stress variation $\Delta \sigma$ as a parameter and the number of cycles $N$ as a variable.

\subsection{Horizon}

To understand the interaction between existing mesocracks and microcracks, the horizon [20] is the key element. It describes the change of the size of obscuration zones of mesocracks in the space versus number of cycles (Figure 2). Lets us consider a microcrack D that may form a mesocrack after $N$ cycles. For each number of cycles $N_{I}$ (with $N_{\text {min }}<N_{I}<N$ ), for all the possible positions of mesocracks, some of them, namely the closest to D, obscure the microcrack location D. This obscured space corresponds to the horizontal bold line in Figure 2 and its size is equal to the size of the obscuration zone $Z_{\text {obs }}\left(N-N_{I}\right)$, where $N-N_{I}$ correspond to the number of cycles of propagation of a mesocrack formed at $N_{I}$ cycles and observed after $N$ cycles. The horizon is the union of all mesocrack positions that obscure D and corresponds to the white zone in Figure 2. Consequently, for a mesocrack to be formed, its horizon should not contain any mesocrack. In the present setting, obscuration occurs at the mesoscopic level because a microcrack shields neither a microcrack nor

a mesocrack, and because only the propagation of mesocracks is taken into 
account in the formation of crack networks.

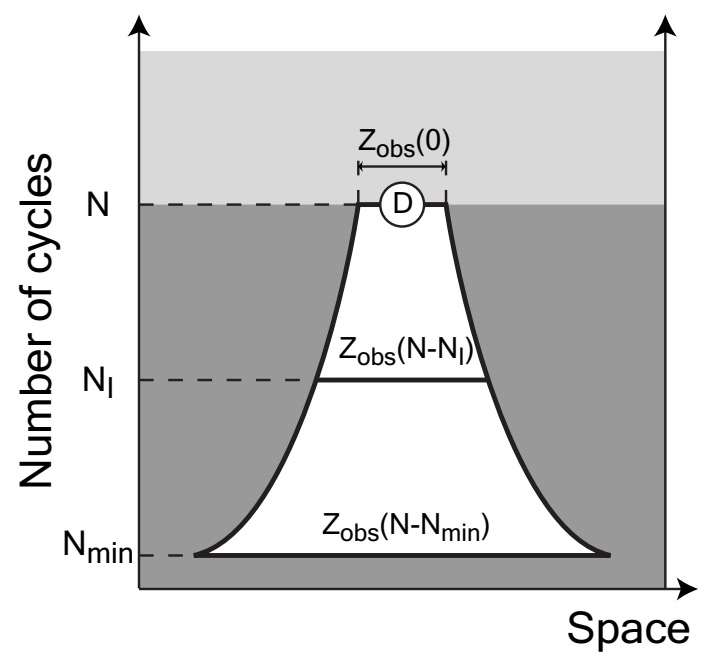

Fig. 2. Horizon of a given site location D.

\subsection{Obscuration Probability}

Apart from shielding effects, the probability of finding $N_{\mu}=\nu$ mesocracks within a uniformly loaded examination domain $\Omega$ is expressed in terms of a Poisson distribution

$$
P\left(N_{\mu}=\nu, \Omega\right)=\frac{\left[\lambda_{t I}(\Delta \sigma, N) Z\right]^{\nu}}{\nu !} \exp \left[-\lambda_{t I}(\Delta \sigma, N) Z\right]
$$

where $Z$ is the size of the domain $\Omega$ so the product $\lambda_{t I}(\Delta \sigma, N) Z$ corresponds to the average number of mesocracks in a domain $\Omega$ loaded for $N$ cycles with a stress variation $\Delta \sigma$, when shielding effects are not considered. The presence of one or more mesocracks in the horizon of a microcrack or a mesocrack induces its obscuration. Hence, one introduces the obscuration probability $P_{\text {obs }}$ that corresponds to the probability of finding one or more mesocracks in 
this horizon

$$
P_{o b s}(\Delta \sigma, N)=1-\exp \left[-\lambda_{t I}(\Delta \sigma, N) \hat{Z}_{o b s}(\Delta \sigma, N)\right]
$$

where $\hat{Z}_{o b s}(\Delta \sigma, N)$ is the measure of the horizon also called the mean obscuration zone [20]

$$
\hat{Z}_{o b s}(\Delta \sigma, N) \lambda_{t I}(\Delta \sigma, N)=\int_{N_{\min }}^{N} Z_{o b s}\left(\Delta \sigma, N-N_{I}\right) \frac{\mathrm{d} \lambda_{t I}}{\mathrm{~d} N_{I}}\left(\Delta \sigma, N_{I}\right) \mathrm{d} N_{I}
$$

$P_{o b s}$ characterizes the degree of damage (i.e., the fraction of obscured zones) of the network. By analyzing its value, the state of cracking in the network can be assessed.

\subsection{Mesocrack Density}

The density of activated mesocracks (i.e., the propagating and stopped mesocracks taking into account shielding effects) is calculated from the formation density $\lambda_{t I}$ and the obscuration probability $P_{o b s}$. This density, denoted by $\lambda_{m}$, corresponds to the density of microcracks that meet all the conditions to form a mesocrack, namely, a number of cycle $N$ greater than the incubation time and the absence of a shielding zone around its location (i.e., this microcrack is not obscured by any mesocrack). The increment of mesocrack density $\lambda_{m}$ is related to that of $\lambda_{t I}$ by

$$
\frac{\mathrm{d} \lambda_{m}}{\mathrm{~d} N}(\Delta \sigma, N)=\frac{\mathrm{d} \lambda_{t I}}{\mathrm{~d} N}(\Delta \sigma, N) \times\left[1-P_{o b s}(\Delta \sigma, N)\right]
$$

The mesocracks that are not obscured by other mesocracks correspond to the active (i.e., propagating) mesocracks. Let $\lambda_{m P}$ denote the propagation density 


$$
\lambda_{m P}(\Delta \sigma, N)=\lambda_{m}(\Delta \sigma, N) \times\left[1-P_{o b s}(\Delta \sigma, N)\right] .
$$

The formed mesocrack density $\lambda_{m}$ may be compared to observations during inspections of zones where, say, thermal crazing is suspected to occur. However, the most crucial information is the active mesocrack density $\lambda_{m P}$ that is critical for the life of a component. This quantity can be estimated only by comparing two different states of cracking. The presented model is now applied to analyze experiments on a 304L austenitic stainless steel.

\section{$3 \quad$ Material and Loading Parameters}

This work considers only one direction for mesocrack formation and propagation on the surface of the material and a propagation in the depth along a direction perpendicular to the surface. A set of semi-elliptical cracks of length $2 a$ and depth $c$ is considered, with $a=c=67 \mu \mathrm{m}$ at crack formation. It is assumed that the shape of the crack remains semi-elliptical, even though the aspect ratio $c / a$ may change with the number of cycles since the propagation on the surface depends on the stress profile $\sigma_{y y}$ along the $z$ direction (Figure 3).

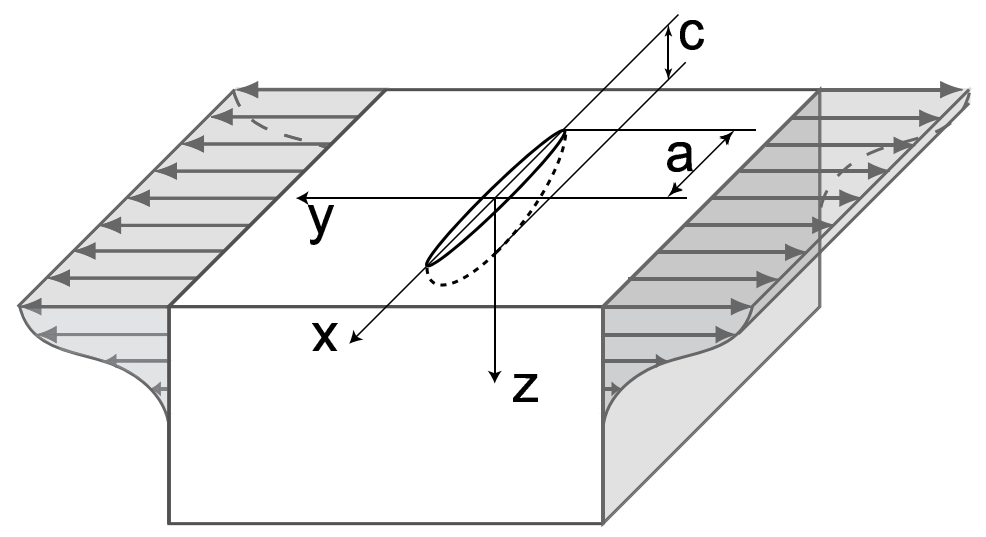

Fig. 3. Crack geometry and loading. 


\subsection{Weibull Parameters}

With a weakest link hypothesis and a power law used to described the microcrack density, a two-parameters Weibull law is retrieved to describe the failure probability for a sample uniformly loaded in the endurance regime (i.e., when $N \rightarrow+\infty)$

$$
P_{F}=P\left(N_{\mu} \geq 1, \Omega\right)=1-P\left(N_{\mu}=0, \Omega\right)=1-\exp \left(-\lambda_{t} Z\right)
$$

The Weibull parameters may therefore be determined by analyzing endurance

data for which the majority of the number of cycles is used in the formation of a mesocrack. An evaluation of the endurance limit of 304L used in the present study has been obtained by following the staircase method [34]. This method, originally proposed by Dixon and Mood [35], gives access to an estimate of the average value $\bar{\sigma}_{\infty}$ and the standard deviation $\overline{\bar{\sigma}}_{\infty}$ of the endurance limit. These two quantities are expressed according to the Weibull model as

$$
\bar{\sigma}_{\infty}=\frac{\sigma_{0}}{\left(\lambda_{0} Z_{e f f}\right)^{1 / m}} \Gamma\left(\frac{1}{m}+1\right)
$$

and

$$
\overline{\bar{\sigma}}_{\infty}=\frac{\sigma_{0}}{\left(\lambda_{0} Z_{e f f}\right)^{1 / m}} \sqrt{\Gamma\left(\frac{2}{m}+1\right)-\Gamma^{2}\left(\frac{1}{m}+1\right)}
$$

where $Z_{\text {eff }}$ is the measure of the effective surface, and $\Gamma$ the gamma function defined by

$$
\Gamma(p)=\int_{0}^{+\infty} t^{p-1} \exp (-t) \mathrm{d} t
$$


Knowing the experimental values of the average and the standard deviation of the fatigue limit distribution, it is possible to determine the Weibull modulus $m$ with the following equation

$$
\left(\frac{\overline{\bar{\sigma}}_{\infty}}{\bar{\sigma}_{\infty}}\right)^{2}=\frac{\Gamma\left(\frac{2}{m}+1\right)}{\Gamma^{2}\left(\frac{1}{m}+1\right)}-1
$$

and then any of the two equations (10) or (11) is used to determine the value

of the last undetermined parameter, namely the scale parameter $\lambda_{0} / \sigma_{0}^{m}$. In this study, $m=20$ and $\sigma_{0}=451 \mathrm{MPa}$ for a value of $\lambda_{0}$ arbitrarily set to $1 \mathrm{crack} / \mathrm{mm}^{2}$.

This analysis shows one of the benefits of the unified framework introduced herein. In the present section, a weakest link hypothesis was made in conjunction with the use of a Poisson point process. A Weibull model is then retrieved, and the parameters of the intensity of the Poisson point process are identical to classical Weibull parameters. Furthermore, the identified parameters will be used later on in a situation in which the weakest link hypothesis does not necessarily apply.

\subsection{Incubation Stress}

As mentioned in the previous part, microcracks may form mesocracks only after a certain number of cycles also called "incubation time." The connection between this incubation time and the formation density is obtained with the incubation stress $\Delta \sigma_{u}(N)$ [Equation (2)]. The difference between the stress variation $\Delta \sigma$ and the incubation stress variation $\Delta \sigma_{u}(N)$ corresponds to the 
fatigue limit $\Delta \sigma_{\infty}$

$$
\Delta \sigma_{\infty}=\Delta \sigma-\Delta \sigma_{u}(N)
$$

To obtain a random number of cycles for mesocrack formation, one only needs to consider the Poisson point process whose intensity is directly related with the previous relationship, via Equation (2). Hence, for a constant mesoscopic stress variation, and a given number of cycles $N$, a direct use of the Poisson point process gives access to the probability of finding $N_{\mu}$ formed mesocracks in the considered zone. Conversely, for a constant mesoscopic stress variation, considering all possible probabilities of finding $N_{\mu}$ formed mesocracks in the considered zone (i.e., from 0 to 1 ), a distribution of the number of cycles to crack formation is retrieved. In uniaxial fatigue tests on a homogeneously loaded specimen, the difference between the number of cycles to form a mesocrack $N_{I}$ and the number of cycles to failure $N_{F}$ of the specimen is a small proportion of this last value, namely

$$
\frac{N_{F}-N_{I}}{N_{f}} \ll 1
$$

In this particular case, considering Equation (14) and a Poisson point process of intensity given by Equation (2), one obtains for a constant mesoscopic stress variation:

- a failure probability for a given number of cycles $N$ (i.e., the probability of finding at least one mesocrack in the considered zone).

- a distribution of the number of cycles to failure of the specimen (i.e., the distribution of the number of cycles for a probability of finding at least one mesocrack ranging from zero to unity). 
The Wöhler curves give access to the variation of stress versus the number of cycles to failure with a given failure probability. The probability curve $P_{F}=0.5$ has been estimated, for 304L stainless steal, by considering the half-life stress variation measured in strain-controlled fatigue tests versus the number of cycles $N_{25}$ (i.e., number of cycles for which a $25 \%$ decrease of maximum stress is obtained). These tests were performed at $165^{\circ} \mathrm{C}$ and with $\dot{\varepsilon}=2 \times 10^{-3} s^{-1}[36,37]$.

Considering a Poisson point process, such a probability curve corresponds to a constant value of its intensity [Equation (2)], and thus to a constant value of $\Delta \sigma_{\infty}$ [Equation (14)]. Therefore, the incubation stress variation $\Delta \sigma_{u}(N)$ may be modeled, say, by a power law [Equation (16)], identified on the change of the difference between the stress variation and the average endurance limit (multiplied by two), versus the number of cycles to failure (Figure 4)

$$
\Delta \sigma_{u}(N)=\sigma_{d} N^{-1 / \eta}
$$

where $\sigma_{d}$ and $\eta$ are material parameters $\left(\sigma_{d}=3.1 \times 10^{5} \mathrm{MPa}\right.$ and $\eta=1.3$ for the considered material). The average endurance limit is estimated for $5 \times 10^{6}$ cycles with staircase stress-controlled fatigue tests. With this power law, it is assumed that $\Delta \sigma_{u}(N \rightarrow+\infty) \rightarrow 0$, which is consistent with the previous analysis. Therefore, the model describes the high cycle fatigue domain (i.e., when $10^{4}<N<+\infty$ ) and the endurance domain (i.e., when $N \rightarrow+\infty$ ) in the same framework. 


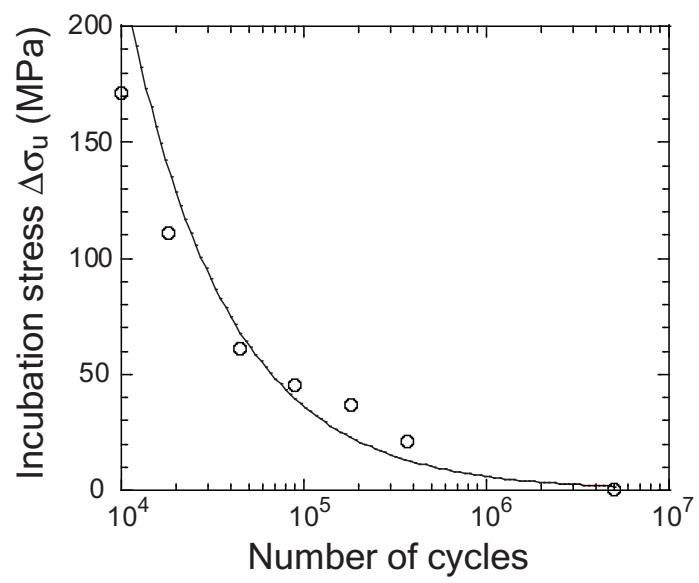

Fig. 4. Identification of the incubation stress $\Delta \sigma_{u}$ as a function of the number of cycles. The open symbols correspond to half-life stress variations measured during strain-controlled tests performed on $304 \mathrm{~L}$ stainless steel at $165^{\circ} \mathrm{C}$, and with $\dot{\varepsilon}=2 \times 10^{-3} \mathrm{~s}^{-1}[36,37]$. The solid line is a power-law fit by least squares minimization.

\subsection{Loading Condition}

In this study, crack networks are due to thermal fatigue and the loading conditions are identified for the SPLASH configuration [25]. The test consists in heating in a continuous way by Joule effect a specimen and to sprinkle distilled water in a cyclic way onto two opposite faces of the specimen. The temperature variation on the surface of the specimen is about $150^{\circ} \mathrm{C}$ and the duration of sprinkling is $0.25 \mathrm{~s}$ each $7.75 \mathrm{~s}$. Maillot [25] carried out thermoelastoplastic finite element calculations to determine the mechanical loading associated with the recorded temperature history. The loading is almost equibiaxial and the variation of stress from the coldest instant $\left(T=T_{\min }\right)$ to the

hottest one $\left(T=T_{\max }\right)$ is estimated at every point of the structure. A $3^{\text {rd }}$ degree polynomial is identified on the calculated stress profile (Figure 5) to model the depth dependence for $z / t \leq 0.16$ of the normal stress 


$$
\frac{\Delta \sigma_{y y}}{\Delta \sigma_{S P L A S H}}=-529.8\left(\frac{z}{t}\right)^{3}+218.3\left(\frac{z}{t}\right)^{2}-28.3\left(\frac{z}{t}\right)+1
$$

where $\Delta \sigma_{S P L A S H}=370 \mathrm{MPa}$ and $t$ is the specimen thickness.

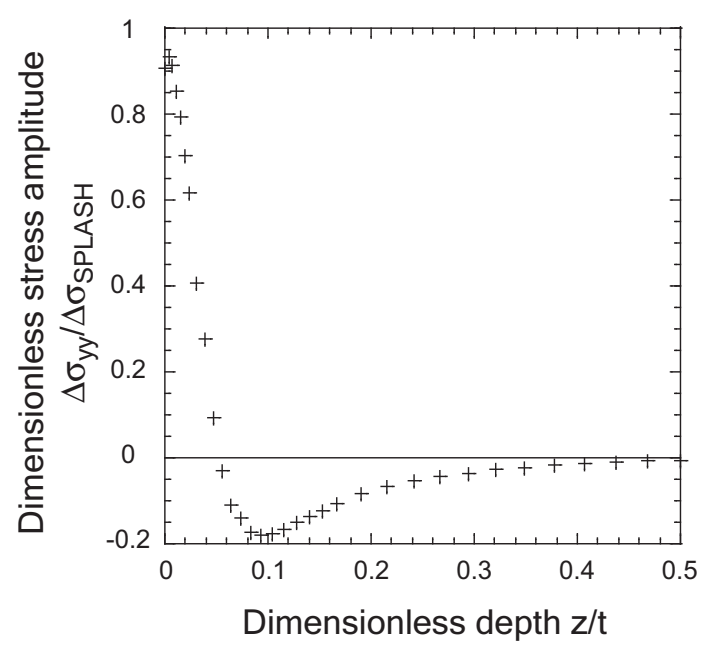

Fig. 5. Computed stress profile.

\subsection{Propagation Law}

Crack networks obtained with the previous stress profile are mainly superficial so the aspect ratio $c / a$ of cracks, which represents the crack shape, is often small (i.e., less than 0.2). The propagation of this type of cracks was studied by Wang and Lambert [38]. It is based on the use of weight functions determined

from finite element results and gives the stress intensity factors $\Delta K_{I A}$ and $\Delta K_{I C}$ associated with semi-elliptical cracks [Figure 6(a)]. A Paris' law is then applied to calculate the crack sizes $a$ and $c$

$$
\frac{\mathrm{d} a}{\mathrm{~d} N}=C\left(\Delta K_{I A}\right)^{p} \quad \text { and } \quad \frac{\mathrm{d} c}{\mathrm{~d} N}=C\left(\Delta K_{I C}\right)^{p}
$$

where $\left.C=3.74 \times 10^{-10} \mathrm{~mm} / \mathrm{MPa} \sqrt{\mathrm{m}}\right)^{-n}$ and $p=4.04$ identified at $320^{\circ} \mathrm{C}$ for the 304L stainless steel considered in this study. Only slight modifications of 
these values are obtained for crack propagation tests performed at room temperature [38] and therefore, these parameters are considered constant during a complete thermal cycle. When the number of cycles is small (i.e., at the beginning of the load history), the crack is propagating on the surface and in-depth [Figure 6(b)]. Yet, as soon as the latter reaches a depth such that the loading becomes compressive, the crack growth rate decreases. The initial conditions for the crack shape are $a=c=67 \mu m$ (i.e., semi circular). Upon loading, the crack shape remains almost circular during one million cycles, then tends quickly towards an elliptic shape during the next million cycles [Figure 6(c)].

\subsection{Obscuration Zone}

By studying the stress field around a penny-crack shaped on a plane submitted to a unidirectional tensile field perpendicular to its propagation direction, a stress relaxation appears around the crack $[39,40]$

$$
Z_{o b s}(N)=S[k a(N)]^{n}
$$

where $S=2$ and $k=1.334$ for a space of dimension $n=1$ [41], and $S=\pi$ and $k=1.428$ for $n=2[42]$. In the present case, the cracks are initiating only on the surface, thus the obscuration is considered only on the surface but one must take into account the effect of mesocrack depth on $Z_{\text {obs }}$. A first approximation is made on this quantity. It is considered that it is equal to the surface of the crack (i.e., the surface of a semi-ellipse of major axis equal to $a$ and minor axis equal to $c$ )

$$
Z_{o b s}(N)=\pi a(N) c(N) .
$$




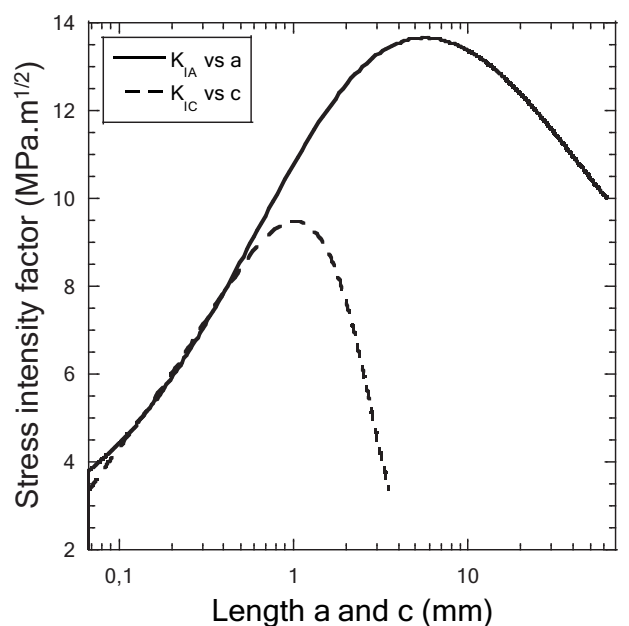

(a) Stress intensity factors vs. crack

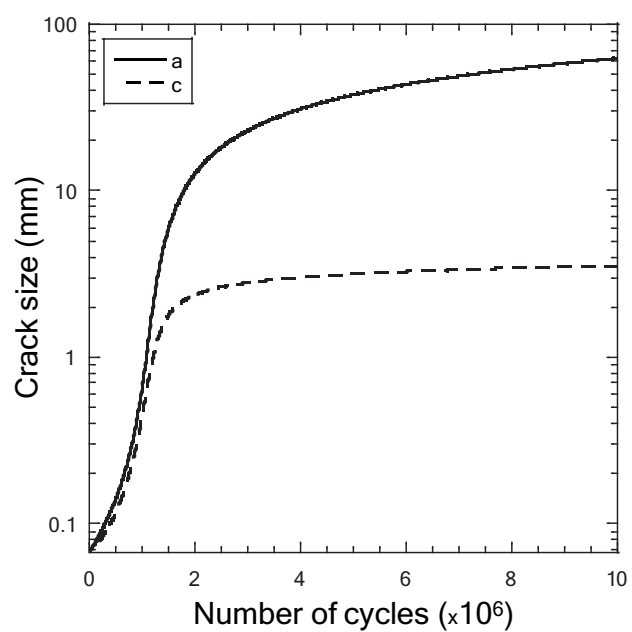

(b) Crack sizes vs. number of cycles length

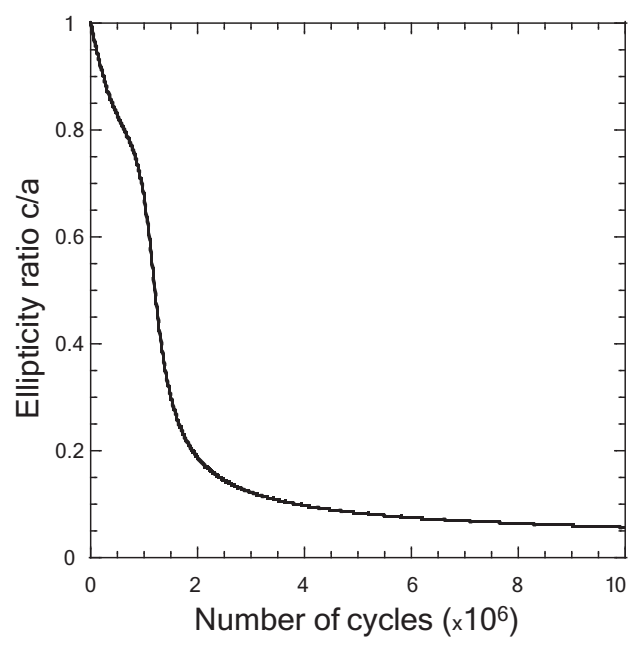

(c) Aspect ratio vs. number of cycles

Fig. 6. Crack propagation features for a SPLASH loading.

The deeper the crack, the larger the obscuration zone on the surface of the specimen.

\section{Results}

In this section, the results of the probabilistic model are presented and compared with results obtained for a more severe loading condition. Some bounds 
are also introduced in order to get analytical expression of the model quantities.

\subsection{SPLASH Conditions}

The SPLASH conditions, presented in Section 3.3, are considered. The first result of this loading condition is the propagation of the first mesocrack described in Section 3.4. These results are regarded as parameters of the probabilistic model, necessary for the calculation of the obscuration probability, the formed and propagating mesocrack densities.

The change of the obscuration probability $P_{\text {obs }}[$ Equation (5)] with the number of cycles is given in Figure 7(a). Figure 7(b) shows the corresponding changes of the formed and propagating mesocrack densities $\lambda_{m}$ and $\lambda_{m P}$. At the beginning, the obscuration probability is equal to zero because of the absence of mesocracks in the sample and thus no obscuration occurs. Both densities are also equal to zero. As soon as the number of cycles reaches the minimum number of cycles (i.e., $N=N_{\text {min }}$ ), when a microcrack forms the first mesocrack, the three quantities begin to increase. When the first mesocrack is formed, an obscuration zone appears in its vicinity and the obscuration probability, which is an increasing function of the size of the obscuration zone, increases. From this moment on, new mesocracks will continue to form with the creation of new obscuration zones and previously formed mesocracks will grow, inducing a growth of their associated obscuration zone. This phenomenon is also accompanied by the arrest of mesocracks that fall into the obscuration zone of other mesocracks and by the impossibility of some microcracks to form new mesocracks because of the relaxation stress field in their vicinity. 
First, the number of arrested mesocracks is less than the number of formed mesocracks, which is confirmed by the increase of the propagating mesocrack density. The increment of arrested mesocracks compared with the increment of new mesocracks is justified by the increasing difference between the two densities [Figure 7(b)]. The maximum of the propagating crack density corresponds to the number of cycles for which the increment of arrested mesocracks becomes greater than that of newly formed mesocracks. From this moment on, the propagating crack density decreases and tends to zero, the obscuration probability tends to 1 (i.e., the studied domain becomes more and more obscured) and the mesocrack density saturates.

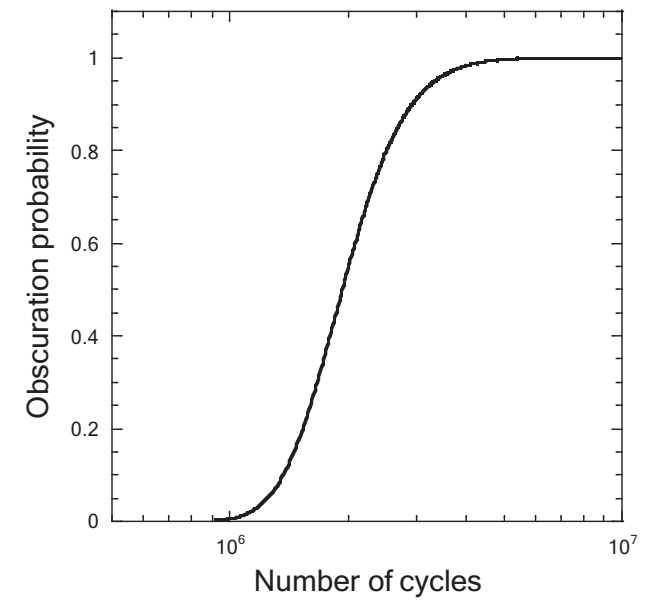

(a) Obscuration probability

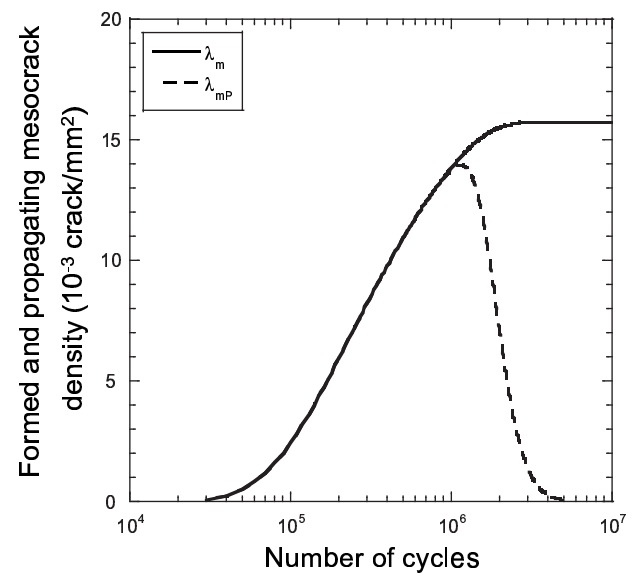

(b) Formed $\lambda_{m}$ and propagating $\lambda_{m P}$ mesocrack densities

Fig. 7. Results of the probabilistic model for a SPLASH loading.

Let us point out that the obscuration probability, the mesocrack and propagating crack densities are related to the surface of the specimen because of the nature of thermal crazing. However, the propagation itself accounts for the in-depth variation of the stress field (see Section 3.3). 


\subsection{Bounds}

Let us derive bounds for the model. An upper bound to the obscuration probability, and lower bounds to the mesocrack and propagating crack densities are given. Let us assume that the crack depth $c$ is equal to a maximum value $c_{\text {max }}$. From Figure 6(a), if one adopts a threshold value for the stress intensity factor $\Delta K_{t h}$ under which the mesocrack is not propagating any more, the maximum crack depth value $c_{\max }$ reads

$$
c_{\max }=c\left(\Delta K_{I}=\Delta K_{t h}\right) .
$$

At the same time, $\Delta K_{I A}$ is almost constant for any value of $a$. This case corresponds to the propagation of a channeling crack [43], i.e., the surface crack propagation velocity $\mathrm{d} \tilde{a} / \mathrm{d} N$ is constant

$$
\frac{\mathrm{d} \tilde{a}}{\mathrm{~d} N}=C\left(\Delta K_{I A \max }\right)^{p}=C \Delta K_{\max }^{p}
$$

where $C$ and $p$ are parameters of the Paris' law. With these assumptions, the half crack size $\tilde{a}$ becomes

$$
\tilde{a}=a_{\text {init }}+C \Delta K_{\max }^{p} \times\left(N-N_{I}\right)
$$

where $a_{\text {init }}$ is the initial size of the mesocrack (here, $a_{\text {init }}=67 \mu \mathrm{m}$ ) and the obscuration probability $\tilde{P}_{o b s}$ reads

$$
\ln \left[1-\tilde{P}_{o b s}(N)\right]=I_{1}+I_{2}
$$

with

$$
I_{1}=\pi c_{\max }\left[a_{\text {init }}+C \Delta K_{\max }^{p} N\right] \times\left[\lambda_{t}\left(N_{\min }\right)-\lambda_{t}(N)\right]
$$


and

$$
\begin{gathered}
I_{2}=-\pi c_{\max } C \Delta K_{\max }^{p} m \lambda_{0}\left(\frac{\Delta \sigma}{\sigma_{0}}\right)^{m} \times \\
\left(\frac{\sigma_{d}}{\Delta \sigma}\right)^{\eta}\left[B\left(\frac{\sigma_{d} N_{I}^{-1 / \eta}}{\Delta \sigma}, 1-\eta, m\right)\right]_{N_{I}=N_{\min }}^{N_{I}=N}
\end{gathered}
$$

where $B$ is the function defined by

$$
B(x, a, b)=\int_{0}^{x} t^{a-1}(1-t)^{b-1} \mathrm{~d} t .
$$

A lower bound to the formed mesocrack density $\tilde{\lambda}_{m}$ is obtained by using Equation (7) in which $P_{o b s}$ is replaced by $\tilde{P}_{o b s}$. Similarly, a lower bound to the propagating mesocrack density $\tilde{\lambda}_{m P}$ is obtained by using Equation (8). The results presented in Figure 8 are obtained with a maximum crack depth $c_{\max }$ equal to $3.5 \mathrm{~mm}$ and they prove that for a mesocrack size always equal to its maximum value, the studied domain is obscured faster than in the case of a normal propagation of mesocracks. The formed mesocrack density increases in the same way as the reference density but slows down earlier and saturates at a lower value. For small numbers of cycles (i.e., $N<10^{5}$ ), the propagating crack density and its bound evolve in the same way but the latter reaches more quickly its maximum value (after $4 \times 10^{5}$ cycles) whereas the normal propagating crack density needs approximatively $10^{6}$ cycles to reach its ultimate level.

\subsection{Comparison with a More Severe Loading}

The previous results were presented in the case of SPLASH conditions. In other cases, such as Fast Breeder Reactors (FBR), the temperature on the in- 


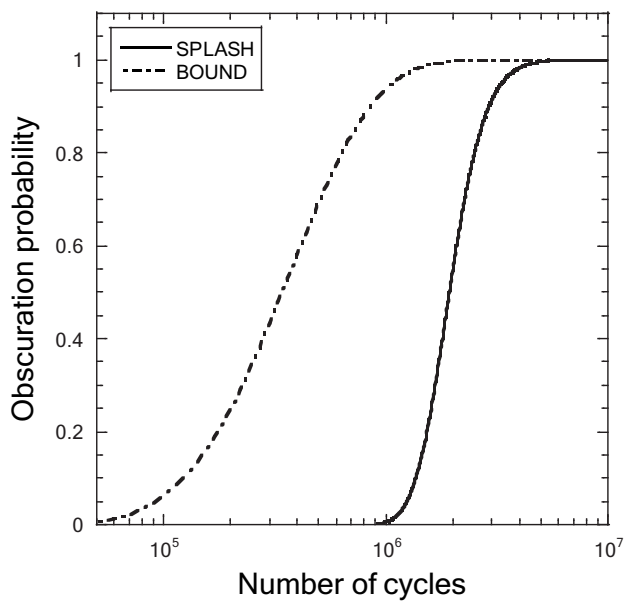

(a)

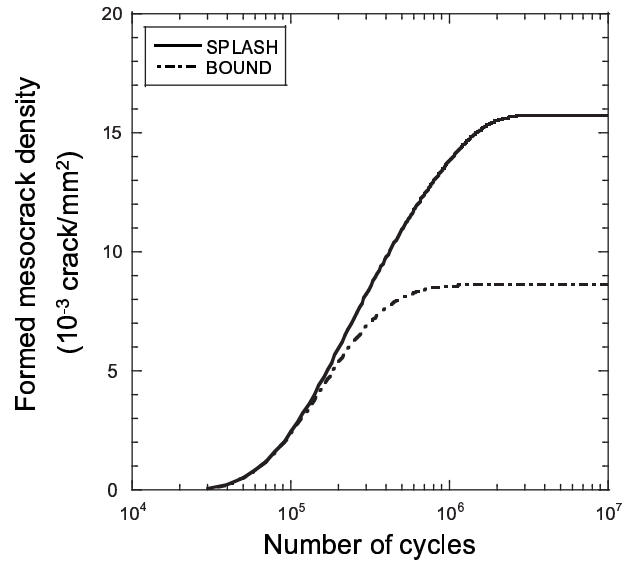

(b)

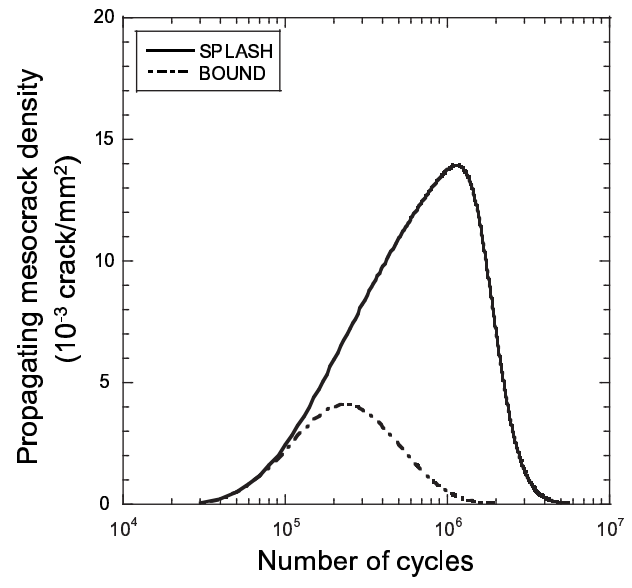

(c)

Fig. 8. Comparison between the model quantities and their respective bounds $\left(c_{\max }=3.5 \mathrm{~mm}\right.$ and $\left.K_{I A}=13.5 \mathrm{MPa} \sqrt{\mathrm{m}}\right)$.

ternal surface of the pipe is higher as well as temperature gradients under the surface. Therefore the stress levels may be larger. In this part, one proposes to apply the model to the case of a higher load level. The associated model is based on Equation (17) where $\Delta \sigma_{S P L A S H}$ is replaced by $\Delta \sigma_{S P L A S H++}$ with $\Delta \sigma_{S P L A S H++}>\Delta \sigma_{S P L A S H}$. The obscuration probability tends to 1 faster in the case of a higher load level [Figure 9(a)]. Moreover, the formed mesocrack density [Figure 9(b)] has higher values compared with those obtained in the SPLASH loading conditions, and its saturation is faster. The propa- 


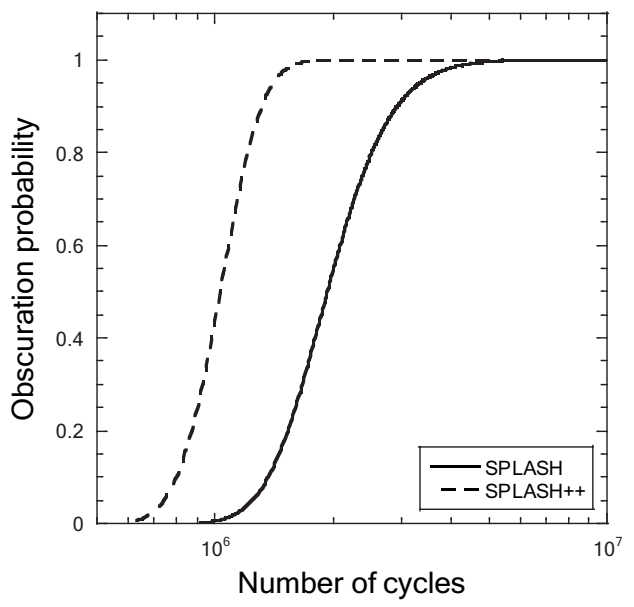

(a)

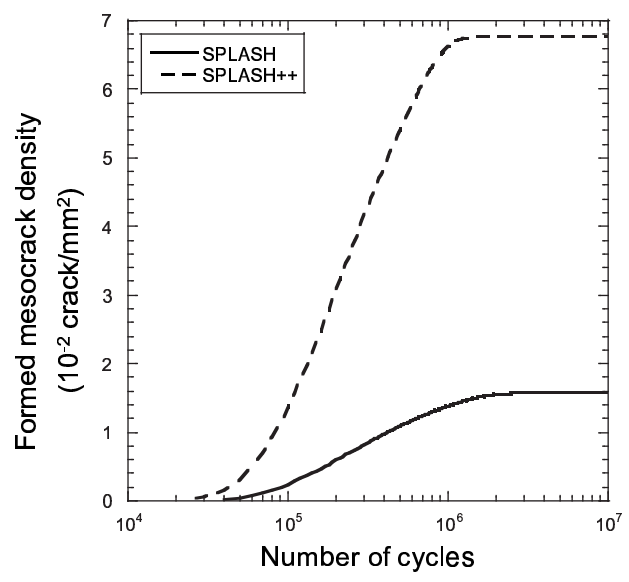

(b)

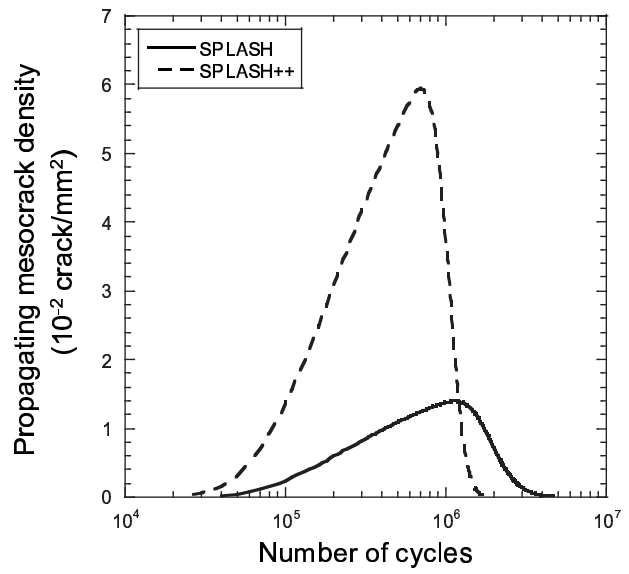

(c)

Fig. 9. Comparison between SPLASH loading and a higher loading (SPLASH++).

gating crack density [Figure 9(c)] increases faster and reaches a maximum value greater than in the case of the SPLASH loading conditions. Its decrease also occurs earlier. Therefore, one concludes that the crack network obtained with a higher load level is denser than that obtained in the normal loading conditions, and its saturation is faster. 


\subsection{Mesocrack Sizes Distribution}

The experimental results [25] give access to the crack network density at several time steps. To obtain the distribution of mesocrack sizes, one should know, at every time, the density of mesocracks that formed at $N_{i}$ cycles and their respective duration of propagation. The density increment of mesocracks formed during the time increment $\mathrm{d} N_{i}$ is given by

$$
\mathrm{d} \lambda_{m}^{*}\left(N_{i}\right)=\frac{\mathrm{d} \lambda_{m}}{\mathrm{~d} N_{i}}\left(N_{i}\right) \mathrm{d} N_{i} .
$$

These mesocracks may be obscured by only larger mesocracks (i.e., mesocracks that were formed before). One associates with $\lambda_{m}^{*}$ the obscuration probability at $N=N_{i}+N_{p}$ of the older mesocracks that formed before $N_{i}$ on the mesocracks that formed at $N_{i}$ cycles and that have potentially propagated for $N_{p}$ cycles

$$
\ln \left[1-P_{o b s}^{i, p}\left(N_{i}, N_{p}\right)\right]=-\int_{N_{\min }}^{N_{i}} \frac{\mathrm{d} \lambda_{t I}}{\mathrm{~d} N_{I}}\left(N_{I}\right) Z_{o b s}\left(N_{p}+N_{i}-N_{I}\right) \mathrm{d} N_{I} .
$$

Thus, the density of mesocracks formed during the time increment $\mathrm{d} N_{i}$ and that have propagated for $N_{p}$ cycles or less is given by

$$
\mathrm{d} \lambda_{s}^{i, p}\left(N_{i}, N_{p}\right)=\frac{\mathrm{d} \lambda_{m}}{\mathrm{~d} N_{i}}\left(N_{i}\right) \mathrm{d} N_{i} \times P_{o b s}^{i, p}\left(N_{i}, N_{p}\right) .
$$

The same type of expression is written for the density of mesocracks formed during the same time increment and that have propagated for $N_{p}+\mathrm{d} N_{p}$ cycles or less. The difference between these two last quantities will give the increment of density of mesocracks formed during $\mathrm{d} N_{i}$ cycles, at $N=N_{i}$ cycles, and that were stopped after a number of cycles of propagation ranging from $N_{p}$ and 
$N_{p}+\mathrm{d} N_{p}$ cycles. This density increment is written as

$$
\mathrm{d} \lambda_{a}^{i, p}\left(N_{i}, N_{p}\right)=\frac{\partial \lambda_{s}^{i, p}\left(N_{i}, N_{p}\right)}{\partial N_{p}} \mathrm{~d} N_{p}
$$

and since $\lambda_{m}$ does not depend on $N_{p}$

$$
\mathrm{d} \lambda_{a}^{i, p}\left(N_{i}, N_{p}\right)=\frac{\mathrm{d} \lambda_{m}\left(N_{i}\right)}{\mathrm{d} N_{i}} \times \frac{\partial P_{o b s}^{i, p}\left(N_{i}, N_{p}\right)}{\partial N_{p}} \mathrm{~d} N_{i} \mathrm{~d} N_{p} .
$$

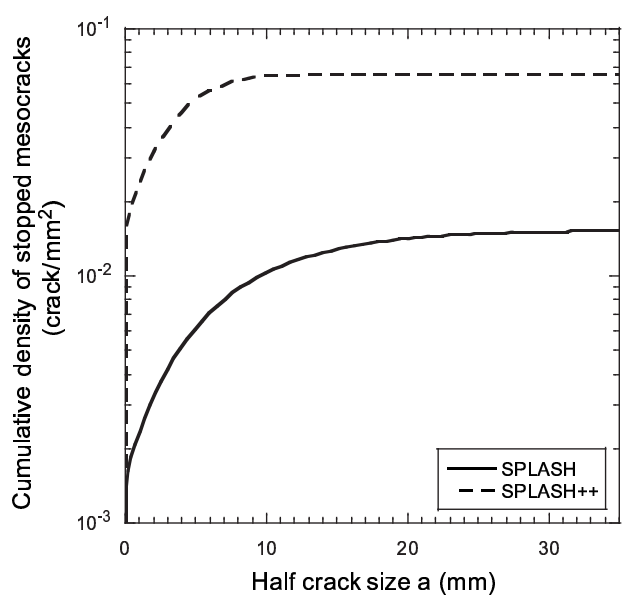

(a)

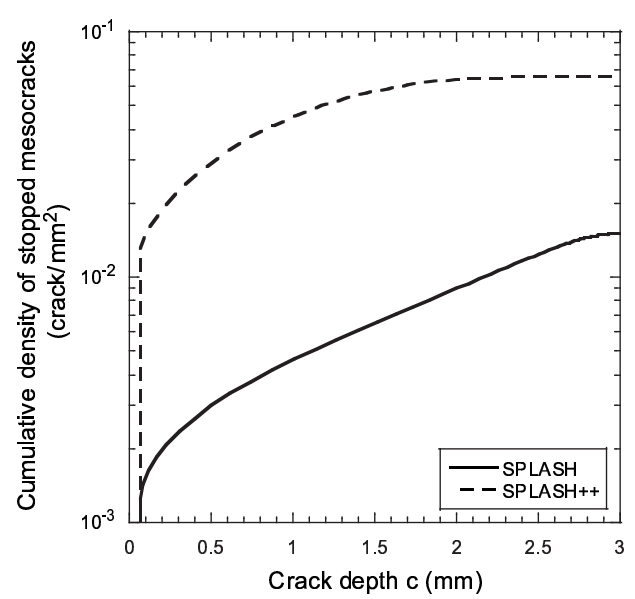

(b)

Fig. 10. Distribution of crack sizes after 5 million cycles for the SPLASH and SPLASH++ loading conditions.

In Figure 10, the cumulated densities of stopped mesocracks after 5 millions cycles are shown as functions of the respective half crack sizes [Figure 10(a)] and depths [Figure 10(b)] for the SPLASH and SPLASH++ loading conditions (in the two cases, after 5 millions cycles, the networks are saturated so the density of stopped mesocracks corresponds to the network density observed in the SPLASH test for example). These curves show that there is no crack with a depth greater than $3 \mathrm{~mm}$ in the SPLASH case, and $2.5 \mathrm{~mm}$ in the SPLASH ++ case (corresponding to a constant value of the cumulative density). Moreover, all stopped mesocracks associated with the SPLASH++ loading condition have sizes on the surface less than $10 \mathrm{~mm}$, whereas the 
stopped mesocracks associated to the SPLASH condition have sizes around 10-20 $\mathrm{mm}$. These results are in qualitative agreement with the experimental results that show crack networks denser and having smaller crack sizes in the case of a stronger loading.

\section{Conclusion}

A probabilistic model was introduced to account for mesocrack formation, propagation and arrest. The obscuration probability characterizes the state of damage of the network. The formed mesocrack density can be compared directly with actual experimental observations of crack networks. The propagating crack density is the key quantity for evaluating the propagation activity in the network, namely, the part of the crack distribution that might be fatal to a structure. All quantities were studied for the particular case of SPLASH loadings. The results of the model are in good qualitative agreement with experimental observations since they present a saturation of crack networks induced by the very small value of the propagating crack density. The arrest of mesocracks in the depth of the material is also accounted for.

In order to find analytical expressions of the model quantities, bounds were proposed by choosing simple laws for the change of crack sizes (i.e., the crack depth and the half-size crack on the surface). These assumptions made it possible to analytically express the formation and propagation of networks made of cracks whose depths are constant and equal to the maximum values observed in the previous case, and for which the surface propagation velocity is maximum. The resulting crack network is less dense than the SPLASH network and saturates faster. The influence of a more severe load level was 
studied and the main conclusion was that the larger the stress variation, the faster the saturation and the denser the crack network with a distribution of smaller crack sizes.

The cases studied herein do not take into account the various possible orientations of the formed and propagating mesocracks (see Ref. [25] on SPLASH tests) and, consequently, the shielding conditions between mesocracks of dif-

ferent orientations. In order to compare quantitatively the predictions of the model and experimental results, the model is currently generalized, by taking into account all the directions of formation and propagation of mesocracks on the surface, as well as their different possible interactions. Last coalescence needs to be accounted for to fully characterize the crack network.

\section{Acknowledgment}

This work was partially funded by Électricité de France R\&D. The authors wish to thank Dr. Saïd Taheri for his support.

\section{References}

[1] M. Mansoor, I. Islam and A. Tauqir, Restricted life of after burner manifold assemblies due to stress raisers, Eng. Fail. Analysis 14 (2007) 1280-1285.

[2] L. Rémy, A. Alam, N. Haddar, A. Köster and N. Marchal, Growth of small cracks and prediction of lifetime in high-temperature alloys, Mat. Sci. Eng. A 468-470 (2007) 40-50.

[3] T. Beck, D. Lohe, J. Luft and I. Henne, Damage mechanisms of cast Al-Si-Mg 
alloys under superimposed thermal-mechanical fatigue and high-cycle fatigue loading, Mat. Sci. Eng. A 468-470 (2007) 184-192.

[4] V. Firouzdor, M. Rajabi, E. Nejati and F. Khomamizadeh, Effect of microstructural constituents on the thermal fatigue life of A319 aluminum alloy, Mat. Sci. Eng. A 454-455 (2007) 528-535.

[5] C. Li, H. Yu, G. Deng, X. Liu and G. Wang, Numerical Simulation of Temperature Field and Thermal Stress Field of Work Roll During Hot Strip Rolling, J. Iron Steel Res. 14 (2007) 18-21.

[6] W. S. Dai, M. Ma and J. H. Chen, The thermal fatigue behavior and cracking characteristics of hot-rolling material, Mat. Sci. Eng. A 448 (2007) 25-32.

[7] A. Persson, S. Hogmark and J. Bergstrom, Simulation and evaluation of thermal fatigue cracking of hot work tool steels, Int. J. Fat. 26 (2004) 1095-1107.

[8] X. Tong, H. Zhou, Z. Zhang, N. Sun, H. Shan and L. Ren, Effects of surface shape on thermal fatigue resistance of biomimetic non-smooth cast iron, Mat. Sci. Eng. A 467 (2007) 97-103.

[9] G. Degallaix, P. Dufrenoy, J. Wong, P. Wicker and F. Bumbieler, Failure mechanisms of TGV brake discs, Key Eng. Mat. 345-346 (2007) 697-700.

[10] C. H. Gao, J. M. Huang, X. Z. Lin and X. S. Tang, Stress Analysis of Thermal Fatigue Fracture of Brake Disks Based on Thermomechanical Coupling, J. Tribol. 129 (2007) 536-543.

[11] X. W. Liu and W. J. Plumbridge, Damage Produced in Solder Alloys during Thermal Cycling, J. Electronic Mat. 36 [9] (2007) 1111-1120.

[12] R. L. J. M. Ubachs, P. J. G. Schreurs and M. G. D. Geers, Elasto-viscoplastic nonlocal damage modelling of thermal fatigue in anisotropic lead-free solder, Mech. Mat. 39 (2007) 685-701. 
[13] S. Taheri, Some advances on understanding of high cycle thermal fatigue crazing, J. Press. Vessel Tech. 129 [3] (2007) 400-410.

[14] O. Ancelet, S. Chapuliot, G. Henaff and S. Marie, Development of a test for the analysis of the harmfulness of a 3D thermal fatigue loading in tubes, Int. J. Fat. 29 (2007) 549-564.

[15] R. Desmorat, A. Kane, M. Seyedi and J.-P. Sermage, Two scale damage model and related numerical issues for thermo-mechanical High Cycle Fatigue, Eur. J. Mech. A/Solids 26 (2007) 909-935.

[16] M. Missirlian, F. Escourbiac, M. Merola, A. Durocher, I. Bobin-Vastra and B. Schedler, Damage evaluation under thermal fatigue of a vertical target full scale component for the ITER divertor, J. Nuclear Mat. 367-370 (2007) 1330-1336.

[17] J.-M. Stephan, F. Curtit, C. Vindeirinho, S. Taheri, M. Akamatsu and C. Peniguel, Evaluation of the risk of damage in mixing zones: EDF R \& D programme, Proceedings Fatigue 2002, 1707-1714.

[18] D. Jeulin, Modèles morphologiques de structures alátoires et changement d'échelle, (thèse d'État, University of Caen, 1991).

[19] R. Gulino and S. L. Phoenix, Weibull Strength Statistics for Graphite Fibres Measured from the Break Progression in a Model Graphite/Glass/Epoxy Microcomposite, J. Mater. Sci. 26 [11] (1991) 3107-3118.

[20] C. Denoual, G. Barbier and F. Hild, A Probabilistic Approach for Fragmentation of Ceramics under Impact Loading, C. R. Acad. Sci. Paris $\mathbf{3 2 5}$ [Série IIb] (1997) 685-691. See also, C. Denoual and F. Hild, A Damage Model for the Dynamic Fragmentation of Brittle Solids, Comp. Meth. Appl. Mech. Eng. 183 (2000) 247-258.

[21] J. Weiss, Endommagement en viscoplasticité cyclique sous chargement multiaxial à haute température d'un acier inoxydable austénitique, (PhD 
dissertation, École Nationale Supérieure des Mines de Paris, 1992).

[22] B. Fedelich, A stochastic theory for the problem of multiple surface crack coalescence, Int. J. Fract. 91 (1998) 23-45.

[23] C. Doudard, S. Calloch, P. Cugy, A. Galtier and F. Hild, A probabilistic twoscale model for high cycle fatigue life predictions, Fat. Fract. Eng. Mat. Struct. 28 (2005) 279-288.

[24] A. Bataille and T. Magnin, Surface damage accumulation in low-cycle fatigue: Physical analysis and numerical modeling, Acta Metall. Mater. 42 [11] (1994) $3817-3825$.

[25] V. Maillot, Amorçage et propagation de réseaux de fissures de fatigue thermique dans un acier inoxydable austénitique de type X2 CrNi 18-09 (AISI 304 L), (PhD dissertation, Université des Sciences et Technologie de Lille, 2003).

[26] D. J. Marsh, A thermal shock fatigue study of 304 and 316 stainless steels, Fat. Engng. Mater. Struct. 4 [2] (1981) 179-195.

[27] C. Robertson, M. C. Fivel and A. Fissolo, Dislocation substructure in 316L stainless steel under thermal fatigue up to $650 \mathrm{~K}$, Mat. Sci. Eng. A $\mathbf{A 3 1 5}$ (2001) 47-57.

[28] C. Robertson and S. Chaise, Bilan des essais de fatigue thermique avec contrainte de traction sur un acier austénitique, (CEA Saclay, technical note, DMN/SRMA/LC2M/NT-2004-2690-A 2004).

[29] O. Ancelet, Étude de l'amorçage et de la propagation des fissures sous chargement thermique cyclique 3D, (PhD dissertation, University of Poitiers, France, 2006).

[30] F. Curtit and J.-M. Stephan, Essais de fatigue thermique sur structures, Proceedings Congrès national Mecamat 2007, 246-255. 
[31] K. J. Miller, Materials science perspective of metal fatigue resistance, Mat. Sci. Tech. 9 (1993) 453-462.

[32] A. M. Freudenthal, Statistical Approach to Brittle Fracture, in: Fracture, H. Liebowitz, ed., (Academic Press, New York (USA), 1968), 591-619.

[33] W. Weibull, A Statistical Theory of the Strength of Materials, (Roy. Swed. Inst. Eng. Res., Report 151, 1939).

[34] L. Vincent and G. Perez, Étude de la fatigue d'un acier inox aux faibles niveaux de chargement. Rapport d'avancement no. 2, (CEA Saclay, technical note, DMN/SRMA/LC2M/NT/2004-2687/A 2004).

[35] W. J. Dixon and A. M. Mood, A method for obtaining and analyzing sensitivity data, J. Am. Statis. Assoc. 43 (1948) 109-126.

[36] M. Mottot and M. Noblecourt, Étude du comportement en fatigue oligocyclique à $165^{\circ} \mathrm{C}$ et $320^{\circ} \mathrm{C}$ du $304 \mathrm{~L}$ pour de faibles niveaux de déformations ( $\varepsilon \leq 1 \%$ ), (CEA Saclay, technical note, DMN/SRMA/NT/2001-2403 2001).

[37] M. Mottot and M. Noblecourt, Étude du comportement en fatigue oligocyclique d'aciers de type Z2 CN 18-10 (304L) à $20^{\circ} \mathrm{C}$ et $300^{\circ} \mathrm{C}$, (CEA Saclay, technical note, DMN/SRMA/NT/2002-2513 2002).

[38] X. Wang and S. B. Lambert, Stress intensity factors for low aspect ratio semi-elliptical surface cracks in finite-thickness plates subjected to nonuniform stresses, Eng. Fract. Mech. 51 [4] (1995) 517-532.

[39] S. M. Seyedi, Formation, propagation et coalescence dans un réseau de fissures en fatigue thermique, (PhD dissertation, École Normale Supérieure de Cachan, 2004).

[40] N. Malésys, M. Seyedi, L. Vincent and F. Hild, On the formation of crack networks in high cycle fatigue, C.R. Mecanique 334 (2006) 419-424. 
[41] B. Widom, Random Sequential Addition of Hard Spheres to a Volume, J. Chem. Phys. 44 [10] (1966) 3888-3894.

[42] J. Quintanilla and S. Torquato, Local volume fraction fluctuations in random media, J. Chem. Phys. 106 [7] (1997) 2741-2751.

[43] J. W. Hutchinson and Z. Suo, Mixed Mode Cracking in Layered Materials, in: Advances in Applied Mechanics, J. W. Hutchinson and T. Y. Wu, eds., (Academic Press, Inc., New York (USA), 1992), 63-191. 